\title{
A Solution for Mobile DTN in a Real Urban Scenario
}

\author{
A. Bujari, C. E. Palazzi \\ Department of Pure and Applied Mathematics \\ University of Padua, Padua - Italy \\ \{abujari|cpalazzi\}@math.unipd.it
}

\author{
D. Maggiorini, C. Quadri, G. P. Rossi \\ Department of Information and Communication Science \\ University of Milano, Milano - Italy \\ \{dario|quadri|rossi\}@dico.unimi.it
}

\begin{abstract}
The growing number of mobile devices equipped with a wireless interface and the end-user trend to shift toward wireless technology is opening new possibilities for networking. In particular, opportunistic communication embodies a feasible solution for environments with scarce or costly infrastructurebased connectivity. In this context we provide a delay-tolerant solution that provides service opportunistic connectivity. Our approach follows the Delay/Disruption Tolerant Network (DTN) paradigm by implementing a store-carry-and-forward communication model among mobile users and buses, the latter embodying carrier entities. A user can delegate the carrier a request which involves Internet access. This request is then forwarded to the bus station's Internet Gateway (IG) in an opportunistic fashion, including both data muling and multi-hop transmission through other buses. Once the request is served at the bus station's IG, the result is opportunistically sent back toward the bus line where the user expects the result. In this paper we present our idea and discuss results obtained through simulations in a realistic urban scenario.
\end{abstract}

Keywords-component; DTN; mobile; opportunistic networking; vehicular network

\section{INTRODUCTION}

The growing availability of personal mobile devices with communication capabilities has boosted the demand for mobile services, which are nowadays typically offered based on connectivity. Yet, connectivity is not always available as infrastructure networks like Internet lack of total coverage of the territory and suffer from what is called the last mile problem [1]. Furthermore, $3 \mathrm{G}$ is not an optimal solution either due to its limited scalability, pull-based service model and interoperation issues among users of competing providers [2]. For this reasons, many researchers are looking today for innovative solutions offering a carrier independent, locationbased, and cheaper than $3 \mathrm{G}$ data distribution. Different types of potential networking environments are investigated, including infrastructure connected networks like cellular networks, mesh networks, vehicular networks, and even entirely disconnected networks of stand-alone mobile devices [3]-[7].

Opportunistic networking, in terms of involving multi-hop Delay/Disruption Tolerant Networking (DTN [8]), represents an interesting alternative as it has no usage costs and could provide service coverage where an infrastructure is not available. Recent trends show that DTNs have gain a lot of popularity in interconnecting mobile users in an ad-hoc fashion and provide service access by leveraging on node mobility to reach infrastructure end-points [9], [10].
While Opportunistic Network management is still a challenge on devices carried by humans due to users' unpredictable movements, this kind of networks has been successfully deployed on Public Transportation Systems (PTSs). Bus-based opportunistic networks are more feasible than their human counterpart because the node mobility is quasi-deterministic: buses move along pre-determined paths approximately following a known schedule. Routing algorithms can then be devised on reasonable assumptions and probabilistic predictions of encounters [11]-[14].

Yet, a crucial and unsolved technical challenge in this scenario is about the scalability of the routing strategies when applied to a larger area, a growing number of lines, and a potentially huge offered load. Since size and shape of bus lines are limited by human and organizational factors, network delivery delay may ramp up exponentially with the covered area due to the increasing number of hops each packet must traverse.

In this context we propose and analyze the performance of Mobile Delay/Disruption Tolerant Network (MDTN): a delay tolerant application platform built on top of a PTS which is able to provide service coverage where Internet is not available [15]. Our software achieves this by implementing a DTN-like, store-carry-and-forward communication model, where a mobile user can delegate a request (e.g., retrieving a web page or sending an email) to a carrier entity (an AP on a bus) and specify on which bus line, later, she/he would like to receive the outcome of her/his request. The request to be served has to reach one of the Internet Gateways (IGs) located at bus line terminals and, finally, the response has to reach the bus line indicated by the users that made the request. To do so, the request is opportunistically forwarded toward the destination bus line by multi-hop transfer among encountering buses. In its trip toward destination, the request can be served by an IG at any traversed line end. From there on, the response will be forwarded toward the destination line, still in an opportunistic, DTN fashion. If no IG is met while the request is in transit, it will be up to the destination line to satisfy the request by reaching its terminal IG. From this point on, the response propagation from the IG toward all buses belonging to the destination will start.

We show in this work the performance trend of our MDTN by means of extensive simulations considering a realistic scenario where carriers are public buses with routes corresponding to actual PTS lines in Milan, Italy, and users are mobile entities owning handheld devices. 
The remainder of this paper is organized as follows. Section II summarizes background information needed to comprehend the applicative domain; Section III gives some insights about MDTN, its modus operandi and architectural design; in Section IV the simulated environment is described and, in Section V, experimental results are discussed. Section VI concludes the paper.

\section{BACKGROUND AND RELATED WORK}

In this section we provide some insights on DTNs, their characteristics, purpose and communication paradigm, and we will outline similar relevant work in the area.

\section{A. Delay/Disruption Tolerant Network (DTN)}

Each network is adapted to particular communication characteristics; the TCP/IP protocol suite [16] has been a great success at interconnecting communication devices across the Internet where connectivity relies primarily on wired links continuously connected in end-to-end, low-delay paths between the sources and destinations. Nowadays, many evolving and potential networks do not confirm to the Internet underlying assumptions. These networks are characterized by intermittent connectivity, long or variable delays, asymmetric data rates, and high error rates.

A DTN, originally thought and conceived for communication in outer space, is an overlay of networks, including the Internet [9]. DTNs achieve interoperability by interfacing between different communication networks characteristics. In providing these functions, DTNs accommodate the mobility and limited power evolving wireless communication devices. They overcome the aforementioned problems by employing a store-carry-and-forward message switching. Whole messages or pieces of such messages are forwarded from a storage place on one node to a storage place on another node, along a path that eventually reaches the destination. To do so, DTN routers need persistent storage for their queues as opposed to Internet routers that use short-term storage provided by memory chips. This can be due to many factors: $(i)$ a communication link to the next hop may not be available for a long time, (ii) one node in a communicating pair may send or receive data much faster or more reliably than the other node, and (iii) a message, once transmitted, may need to be retransmitted if an error occurs at an upstream link, or if an upstream node declines acceptance of a forwarded message.

All the aforementioned functionalities are provided in DTNs by adding a new protocol layer called the Bundle Layer (BL) [17]. The Bundle Layer (BL) sits over the specific Transport Layer of a network and aims to link together different and heterogeneous networks, allowing message transmissions (bundles) between and among them (Fig. 1).

Our idea is to build a DTN between mobile users and carriers who communicate through Wi-Fi instead of UMTS or other related technologies whose usage is associated to a cost. We leverage on carrier mobility to reach infrastructure endpoints which have Internet connectivity.

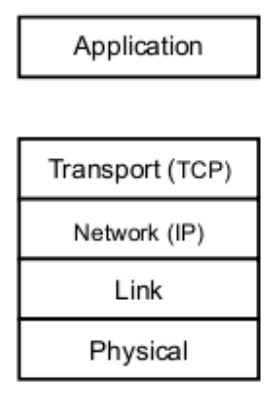

Internet Layers

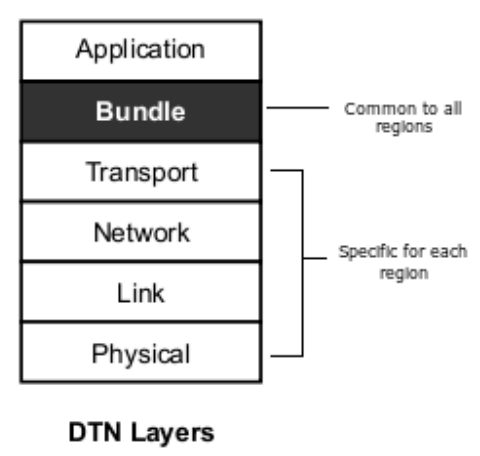

Figure 1. DTNs Bundle Layer.

\section{B. Related Work}

Despite the fact DTNs were introduced in 2003 as an outer space architectural paradigm, many efforts have been devoted by the scientific community to devise reliable and efficient distribution strategies for these kinds of architectures for terrestrial applications [18]-[21].

The first contributions focused on rural environments in developing regions where a number of villages are spread over a large territory linked by buses [13], [14], [22]. The common goal of all those projects was to provide network access for elastic non real-time applications so that the local population may enjoy basic Internet services (e.g., e-mail and non-real time web browsing).

Campus bus networks (e.g., [11], [12], [23]) are designed to serve students and faculties who commute between colleges or from/to nearby towns. These kinds of services are usually characterized by a relatively small number of nodes when compared to a fully fledged urban environment. The main contribution in this direction is represented by [11] and [23], where five colleges are linked with nearby towns and to one another over an area of 150 square miles. On this same bus network, a system of throwbox nodes [24] was deployed to enhance the capacity of the DTN.

Scaling up in terms of number of nodes, we find urban environments where a considerable number of lines are densely deployed to enable people to commute inside a city. Bus networks in urban environment (e.g., [25]-[28]) are usually characterized by many contact opportunities and frequent contacts. In [25], authors propose a commercial application called Ad Hoc City. Based on a multi-tier wireless ad-hoc network routing architecture it provides elastic Internet access by means of Access Points, which are responsible for a geographical area. The proposed system targets generalpurpose wide area communication. Messages from mobile devices are carried to the AP and back using an ad-hoc backbone that exploits buses. The authors verified the validity of the proposed approach against real movement traces by King County Metro bus system in Seattle, WA. Using the same real data as for [25], the authors of [26] propose a cluster-based routing algorithm for intra-city message delivery. In [26] an efficient large-scale clustering methodology is devised: nodes are clustered based on the basis of encounter frequency while multi-copy forwarding takes place between members of the same cluster hosting the destination node. To lessen the 
overhead effect of having multiple copies in the network, the authors of [27] model forwarding as an optimal stopping rule problem. The contribution from [28] uses data from the public transportation system of Shanghai to test the performance of a single-copy forwarding mechanism. This is a probabilistic routing strategy where probabilities are related to intra-contact times as in [29].

\section{MDTN}

MDTN will be outlined in this section. First we will describe its modus operandi; then, we will give an overview of the associated protocol.

\section{A. Modus operandi}

Observing Fig. 2 we can see that there are three main entities involved in the MDTN architecture; they are: $(i)$ the MDTN client or a mobile user operating a smartphone and (ii) the MDTN server or the bundle carrier which locally stores users requests, tries to accomplish them when Internet connection is available, and (iii) the IG, deployed at each bus terminal, which has Internet connectivity.

Once the user gets on board of the carrier, it establishes a connection with the on board hosted server and sends it a request. The request consists of a specific required content and a destination line the user is going to take later on. The goal of MDTN is to provide the user with the desired content on the specified destination line.

Delivery of response on the required destination line is performed in the following manner:

A. The carrier which has the request encounters during its trip a carrier belonging to a valid next-hop line, and forwards the request to it. It is now up to the new carrier to satisfy the request if it reaches its end of line and hence an IG (Fig. 2).

B. The carrier which has the request reaches its terminal, and is able to fulfill the request by itself; from now on the result will be forwarded toward the destination line.

Of course, the request could reach a bus of the destination line before being served by any IG. In this case that bus will transport the request till its $\mathrm{IG}$, the request will be served and the response made available.

Moreover, as there are many carriers running a destination line, an additional step is required: dissemination of content among carriers of the same line. Regardless of the delivery scenario, a carrier of the destination line will eventually reach its terminal carrying either the request to fulfill or the response produced during forwarding. The response will be stored in the terminal IG that, later on, will take care to forward the output toward all other carriers of the same line for delivery.

Of course, an alternative solution could be to transfer the result to the IG of the destination line via wired infrastructure. We are not going here to tackle on this approach for a number of reasons. First, we are interested in understanding the upper bound imposed by our software platform. Second, breaking the end-to-end paradigm could lead to scalability issues when increasing the number of lines or the size of the city. Third, this way we are adopting a triangular routing where the first encountered IG and the IG of the destination line can be associated to the home and foreign agents respectively; this way, services may take advantage from some compatibility with mobile IP.

The delivery process is entirely transparent to the client. The client has an established connection with one MDTN server and is notified for the request outcome when/if the request has been accomplished. Obviously, the MDTN client can disconnect/connect from/to the MDTN server at any time and the response will be forwarded next time they pair with each other. It is also important to note that the devised system could provide the user with both pull/push style services.

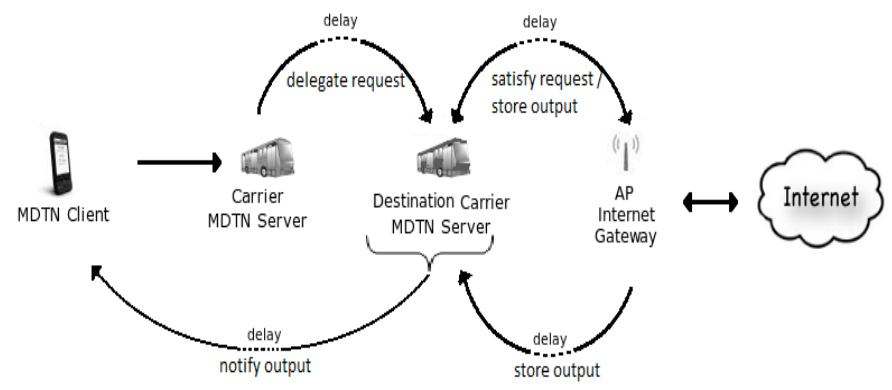

Figure 2. MDTN system orchestration

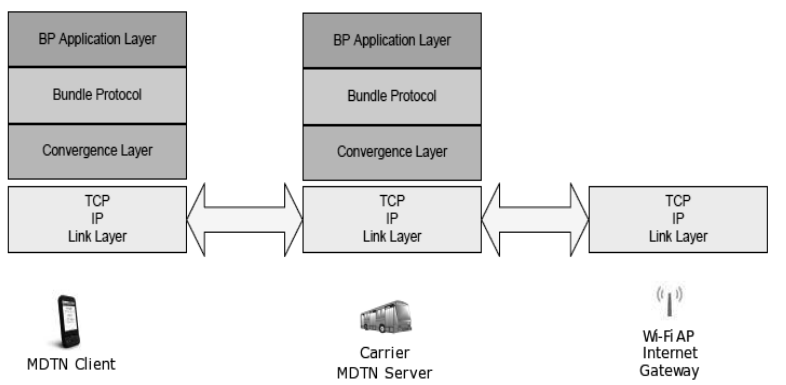

Figure 3. MDTN protocol stack.

\section{B. MDTN protocol}

The MDTN-client can be in the following two possible states:

- online: when he is connected to the MDTN server;

- offline: otherwise.

On the other hand, the MDTN-server can be in the following two states:

- gathering: the server does not have Internet connectivity but it is actively receiving and storing user requests, and eventually forwarding stored information previously gathered for other users;

- digesting: the server reaches an IG and is able to accomplish (digest) pending user requests (gathering mode is still available). 
Both these entities need to implement and use the same communication protocol (Fig. 3), which is a slightly modified version of the Bundle Protocol. It is important to note that both of them implement a BP-Application Layer as it is required and application specific. Also, inside a DTN, every node that is able to send and receive bundles is called bundle node, and is characterized by the presence of three fundamental components:

- BPA (BP-Application Layer): is the Bundle Layer services supplier, which allows higher levels to communicate through the DTN;

- CLA (Convergence Layer Adapter): is the adapter which allows the Bundle Overlay to be placed over various physical networks that can work with different Transport Protocols (like TCP). There can be more than one adapter for each node;

- $\quad$ AA (Application Agent): is the component that uses the BPA for communications purpose.

\section{Simulation ENVIRONMENT}

In this section we overview the simulation settings we used for experiments. First, we describe the urban environment used for simulation and then we provide details about the specific parameters used for simulation.

All simulations have been performed using URBeS (Urban Routing Backbone Simulator): an ad-hoc simulator presented and validated in [30].

\section{A. Urban Environment}

The urban environment we used to evaluate our proposal is the city of Milan (Italy) and its PTS. Milan is a medium size town (typical for many European cities) and its PTS is a complex system extending above and below ground. Due to the underground aquifer and archaeological remains, the subway system is relatively underdeveloped while the ground transportation system spans 69 lines over 49 square miles for a total paths length of 683 miles (13.85 miles for every square mile). The overall city structure is clearly not Manhattan-like due to the adaptation to the old Roman historical center and the progressive annexing of small peripheral towns in the main city body. With this kind of topology, crosses between bus lines occur at any time, there is no constant space between intersections, and streets do not run parallel one to each other for very long.

While on Manhattan-like topologies it is easy to forecast node contacts, the structure we address here is more challenging but represents a realistic case study for urban-wide services simulation.

\section{B. Simulation Parameters}

In our experiments we considered IEEE 802.11b technology. Available bandwidth is $11 \mathrm{Mpbs}$ and the radio range is $100 \mathrm{~m}$. Transmission is accounted using a token bucket model. Communication takes urban canyons into account; we consider only line-of-sight contacts.
Simulation starts at 4 a.m. (the first bus starts its trip at 5 a.m.) and stops at 8 a.m. of the following day (the last bus going out of service around 6 a.m.). All buses departing before 5 a.m. are considered as on duty during the night and not in the morning.

Data traffic generation is performed continuously during working hours: from 8 a.m. to 8 p.m. During simulations, requests are generated at a constant rate for every operating bus. Each bus receives 10 requests per hour as long as it is in service, even when waiting for the next departure at the end of the line. Each request will require for the response to be directed to a distinct, randomly chosen, bus line. To emulate a typical web-based traffic model each request has a size of $1 \mathrm{~KB}$, while responses are $64 \mathrm{~KB}$.

When a packet is generated it is placed in the bus local storage until forwarding becomes possible. When an encounter takes place all stored packets are checked for forwarding and transmitted in accordance with the adopted routing policy if the encounter matches a positive opportunity. Bandwidth is accounted using a token bucket mechanism while buffer space availability is simply checked before transmission. In case of contention a first-in-first-out policy is applied. The size of the application buffer on board at every bus has been set to $1 \mathrm{~GB}$.

As for the routing policy we adopted a link state approach based on minimum hop metric. This approach is feasible because all bus routes are known in advance and do not change without notice during the day; thus, a line-connection matrix can be built and shared among all buses. All paths are calculated minimizing the number of hops required to reach the destination, just like a human traveler will do.

When a bus reaches the end of the line it may or may not queue up and wait for another scheduled departure. If the bus stays in line it will hold all its data and will keep accepting requests from the surroundings. If, on the other hand, the bus leaves service all content will be pushed to the first bus waiting in line. If there is no bus (because there are no more scheduled departures) all the stored packets are dropped and packets are considered lost.

\section{EXPERIMENTAL RESULTS}

The metrics we are considering to evaluate system performance are response delivery time and rate. From these metrics, we are able to understand which kinds of services are meaningful to be provided from the MDTN platform.

Response delivery time is distributed as depicted in Fig. 4. More than $60 \%$ of the requests get satisfied in 2 to 4 hours with a long queue (median of 3.06 hours, see also Tab. I); whereas it takes up to 19 hours for a very limited number of requests. Under these lenses, the system could be actually used for news retrieval, delay-tolerant web browsing, and distribution of information regarding local events. 


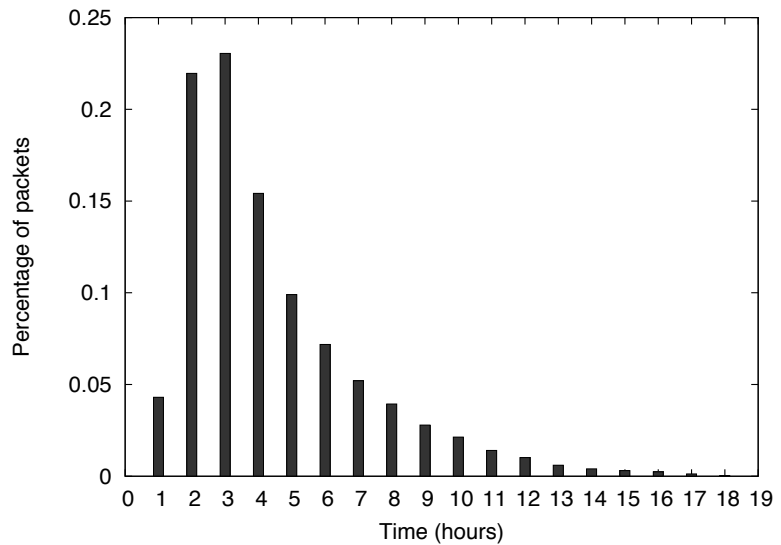

Figure 4. Histogram of response delivery time.

On the other hand, a completely different profile comes out if we consider only the time to retrieve the response (fulfill time in Tab. I). In this case we have an average of 50 minutes, giving a clear indication that the majority of time is spent forwarding the result toward the destination line.

The above behavior is strongly suggesting that pre-fetch mechanisms and caching strategies will help a lot to improve system performance.

TABLE I. SYSTEM PERFORMANCE INDEXES

\begin{tabular}{|l|c|c|c|}
\hline & Average & Median & Std. dev. \\
\hline Fulfill time (hours) & 0.82 & 0.67 & 0.64 \\
\hline Delivery time (hours) & 3.92 & 3.06 & 2.72 \\
\hline Delivery rate (\%) & 70.62 & 70.74 & 0.34 \\
\hline
\end{tabular}

From Fig. 5 we can observe the traffic profile during a day. Data delivery rate is $70.62 \%$ (see also Tab. I). Packet loss starts around midnight due to many lines going out of service thus creating an extremely partitioned network. The indication here is that data loss is due to PTS timetable rather than system failure; a smarter routing strategy, with shorter delivery time, will for sure improve the situation.

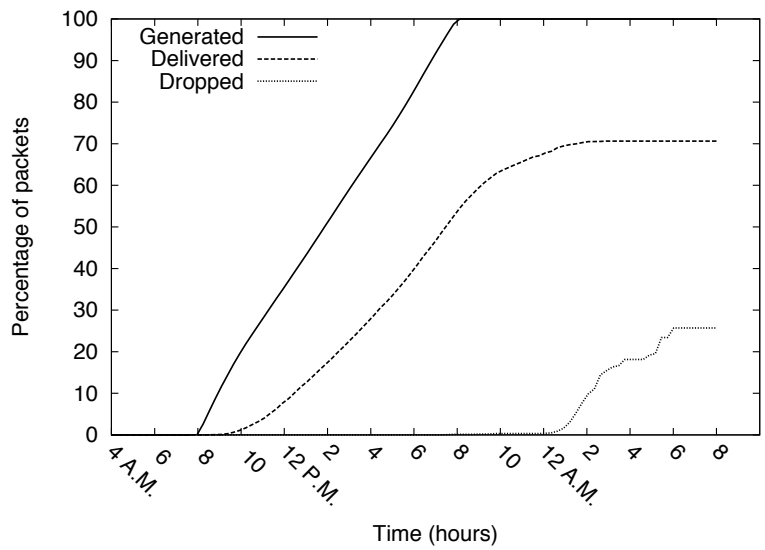

Figure 5. Traffic generation and delivery profiles.

From simulations, an average of $87 \%$ of requests is fulfilled during routing and not at the destination line, suggesting that the performance issues are coming from the routing policy based on a minimum hop metric rather than from traffic congestion. Reducing the delivery time by means of a smarter routing policy will allow to increase the delivery rate and to provide loose real-time services such as short messaging and twitter-like microblogging.

As it is, the MDTN platform can already be effectively used for dissemination of push-based advertisements dissemination and non-critical data retrieval.

\section{CONCLUSION AND FUTURE WORK}

In this paper we studied MDTN, a delay tolerant application platform able to provide service connectivity where coverage is lacking or its access is attributed to a cost (e.g., $3 \mathrm{G}$ cellular networks). Differently from a previous work, we applied it to a real city environment, extending the protocol modus operandi to a more complex and realistic usage scenario. By means of simulation we demonstrated that MDTN could be a viable solution for push-based advertisements dissemination and noncritical data retrieval.

As future extensions for this work we are planning to address the adoption of a more sophisticated and dedicated, routing strategy as well as the evaluation of content caching and data pre-fetch to improve performances.

\section{REFERENCES}

[1] A. Qureshi, J. Carlisle, J. Guttag, "Tavarua: Video Streaming with WWAN Striping", in Proc. of ACM Multimedia 2006, Santa Barbara, CA, USA, Oct 2006.

[2] J. Dedrick, V. Gurbaxani, K. Kraemer, "Information Technology and Economic Performance: A Critical Review of the Empirical Evidence", ACM Computing Surveys, vol. 35, no. 1, pp. 1-28, Mar 2003.

[3] C. E. Palazzi, "Buddy-Finder: A Proposal for a Novel Entertainment Application for GSM", in Proc. of the 1st IEEE International Workshop on Networking Issues in Multimedia Entertainment (NIME'04), GLOBECOM 2004, Dallas, TX, USA, Nov 2004.

[4] C. E. Palazzi, A. Bujari, "A Delay/Disruption Tolerant Solution for Mobile-to-Mobile File Sharing", in Proc. of IFIP/IEEE Wireless Days 2010, Venice, Italy, Oct 2010.

[5] A. Hamidian, C. E. Palazzi, T. Y. Chong, J. M. Navarro, U. Körner, M. Gerla, "Deployment and Evaluation of a Wireless Mesh Network", in Proc. of the Second IARIA/IEEE International Conference on Advances in Mesh Networks (MESH 2009), Athens, Greece, Jun 2009.

[6] A. Amoroso, G. Marfia, M. Roccetti, "Going Realistic and Optimal: A Distributed Multi-Hop Broadcast Algorithm for Vehicular Safety", Computer Networks, Elsevier, vol. 55, n. 10, pp. 2504-2519, Jul 2011.

[7] M. Roccetti, G. Marfia, A. Amoroso, "An Optimal 1D Vehicular Accident Warning Algorithm for Realistic Scenarios", in Proc. of IEEE Symposium on Computers and Communications (ISCC'10), Riccione, Italy, Jun 2009

[8] S. Burleigh, K. Fall, V. Cerf, R. Durst, K. Scott, H. Weiss, L. Torgerson, A. Hooke, "Delay Tolerant Networking - An Approach to Interplanetary Internet", IEEE Communications Magazine, vol. 41, no. 6, pp. 128-136, Jun 2003.

[9] L. Pelusi, A. Passarella, M. Conti, "Opportunistic Networking: Data Forwarding in Disconnected Mobile Ad Hoc Networks," IEEE Communication. Magazine., vol. 44, no. 11, pp. 134-141, Dec 2006.

[10] S. Gaito, D. Maggiorini, E. Pagani, P. G. Rossi, "Distance Vector Routing for Public Transportation Vehicular Networks: Performance Evaluation on a Real Topology", in Proc. of IFIP/IEEE Wireless Days 2009, Paris, France, Dec 2009. 
[11] X. Zhang, J. Kurose, B. N. Levine, D. Towsley, H. Zhang, "Study of a Bus-Based Disruption-Tolerant Network: Mobility Modeling and Impact on Routing", in Proc. of ACM MobiCom'07, Montrèal, QC, Canada, Sep 2007.

[12] A. Balasubramanian, B. Levine, A. Venkataramani, "DTN Routing as a Resource Allocation Problem”, in Proc. of ACM SIGCOMM'07, Kyoto, Japan, Aug 2007.

[13] A. Pentland, R. Fletcher, A. Hasson, "DakNet: Rethinking Connectivity in Developing Nations", IEEE Computer, vol. 37, no. 1, pp. 78-83, Jan 2004.

[14] M. Demmer, K. Fall, "DTLSR: Delay Tolerant Routing for Developing Regions", in Proc. of the 2007 ACM Workshop on Networked Systems for Developing Regions (NSDR'07), Kyoto, Japan, Aug 2007.

[15] C. E. Palazzi, A. Bujari, S. Bonetta, G. Marfia, M. Roccetti, and A. Amoroso, "MDTN: Mobile Delay/Disruption Tolerant Network", in Proc. of the 20th IEEE International Conference on Computer Communications and Networks (ICCCN 2011) - 7th IEEE Workshop on Networking Issues in Multimedia Entertainment (NIME 2011), Maui, HI, USA, Aug 2011.

[16] W.R.Stevens, TCP/IP Illustrated, Volume 1- The Protocols, AddisonWesley, Apr 2000.

[17] K. Scott, S. Burleigh, "Bundle Protocol Specification" draft-irtf-dtnrgarch-02.txt, Jul 2004.

[18] S. Jain, K. Fall, R. Patra, "Routing in a Delay Tolerant Network", in Proc. of ACM SIGCOMM '04, Portland, OR, USA, Aug-Sep 2004.

[19] Q. Yuan, I. Cardei, J. Wu, "Predict and Relay: An Efficient Routing in disruption Tolerant Networks", in Proc. of ACM international Symposium on Mobile Ad Hoc Networking and Computing (MobiHoc'09), New Orleans, LA, USA May 2009.

[20] S. Nelson, M. Bakht, R. Kravets, "Encounter-Based Routing in DTNs", in Proc. of IEEE INFOCOM 2009, Rio de Janeiro, Brazil, April, 2009.

[21] A. Lindgren, A. Doria, O. Schelén, "Probabilistic Routing in Intermittently Connected Networks", in Proc. of ACM SIGMOBILE Mob. Comput. Commun. Rev. vol. 7, no. 3, pp. 19-20, Jul 2003.
[22] C. T. De Oliveira, R. B. Braga, D. M. Taveira, N. C. Fern, O. C. M. B. Duarte, "A Predicted-Contact Routing Scheme for Brazilian Rural Networks", Electrical Engineering Program, COPPE/UFRJ, 2008.

[23] J. Burgess, B. Gallagher, D. Jensen, B. N. Levine, "MaxProp: Routing for Vehicle-Based Disruption-Tolerant Networks", in Proc. of IEEE Infocom 2006, Barcelona, Spain, Apr 2006.

[24] N. Banerjee, M. D. Corner, B. N. Levine, "Design and Field Experimentation of an Energy-Efficient Architecture for DTN Throwboxes", IEEE/ACM Transactions on Networking, vol. 18, no. 2, pp. 554-567, Apr 2010.

[25] J. Jetcheva, Y.-C. Hu, S. PalChaudhuri, A. Saha, D. Johnson, "Design and Evaluation of a Metropolitan Area Multitier Wireless Ad Hoc Network Architecture", in Proc. of 5th IEEE Mobile Computing Systems and Applications 2003 (WMCSA 2003), Monterey, CA, USA, Oct 2003.

[26] S. Ahmed, S. Kanhere, "Cluster-Based Forwarding in Delay Tolerant Public Transport Networks", in Proc. of 32nd IEEE Conference on Local Computer Networks (LCN 2007), Dublin, Ireland, Oct 2007.

[27] C. Liu, J. Wu, "An Optimal Probabilistic Forwarding Protocolin Delay Tolerant Networks", in Proc. of the 10th ACM International Symposium on Mobile Ad Hoc Networking and Computing (MobiHoc'09), New Orleans, LA, USA, May 2009.

[28] M. Sede, L. Xu, L. Da, W. Min-You, L. Minglu, S. Wei, "Routing in Large-Scale Buses Ad Hoc Networks", in Proc. of IEEE Wireless Communications and Networking Conference 2008 (WCNC 2008), Mar-Apr 2008.

[29] K. Tan, Q. Zhang, W. Zhu, "Shortest Path Routing in Partially Connected Ad Hoc Networks", in Proc. of IEEE Global Telecommunications Conference 2003 (GLOBECOM 2003), San Francisco, CA, USA, Dec 2003.

[30] S. Gaito, D. Maggiorini, and G. P. Rossi, "Leveraging Bus Mobility to Enable Communications in Urban Areas," Jul. 2011, arXiv:1107.4526v1 [cs.NI]. 